\title{
Microring resonator-coupled waveguide crossings in silicon nitride: waveguide width tuning
}

\author{
Shengmei Zheng, Chung Yan Fong and Andrew W. Poon \\ Department of Electrical and Electronic Engineering, The Hong Kong University of Science and Technology, \\ Clear Water Bay, Hong Kong SAR, China \\ Tel: (852)-2358-7905, fax: (852)-2358-1485, email: eeawpoon@ust.hk
}

\begin{abstract}
We report high refractive-index contrast microring resonators laterally coupled with waveguide crossings as add-drop filters in silicon nitride. We demonstrate tuning of resonance wavelengths, coupling efficiency, on-off ratio, and finesse by varying the waveguide width.
\end{abstract}

OCIS codes: (230.5750) Resonators; (230.3990) Microstructure devices

High refractive-index contrast microresonators coupled with submicrometer waveguides have been attracting considerable interest for high-density photonic integrated circuit applications [1]. Microresonators, such as microring resonators [2], which are input and output-coupled with parallel waveguides in Group III-V [3] and silicon-based material systems [4] have long been studied for add-drop filter applications. In order to attain large-scale integrated optical cross-connects, microring resonators coupled with orthogonal waveguide crossings have also been proposed and demonstrated on compound glass substrates by vertical coupling [5].

In this summary, we report for the first time to our knowledge, an experimental demonstration of high refractive-index contrast microring resonators laterally coupled with submicrometer waveguide crossings in silicon nitride. Our initial experiments reveal that resonances can be efficiently transferred from the input-coupled waveguide to the crossed waveguide through the side-coupled microring. We demonstrate that resonance wavelengths, coupling efficiency, on-off ratio, and finesse can be tuned by varying the widths of crossing and ring simultaneously.

In our experiment, microring resonators with $50 \mu \mathrm{m}$ outer-diameter are laterally coupled with submicrometer waveguide crossings whose widths vary from $0.7 \mu \mathrm{m}$ to $0.4 \mu \mathrm{m}$. The microring has the same width as the coupled waveguide crossing. The submicrometer air-gap separation between the waveguide and the microring is about $0.4 \mu \mathrm{m}$. We employed standard silicon microfabrication processes as detailed in [6]. The device is comprised of a $1.1 \mu \mathrm{m}$ low-stress silicon nitride film (refractive index $\approx 2$ ) on a $1.5 \mu \mathrm{m}$ silica under-cladding. The device patterns were defined by photolithography and transferred onto the nitride layer by plasma etching. The etched height is about $0.92 \mu \mathrm{m}$ and the device is air-clad.

(a)

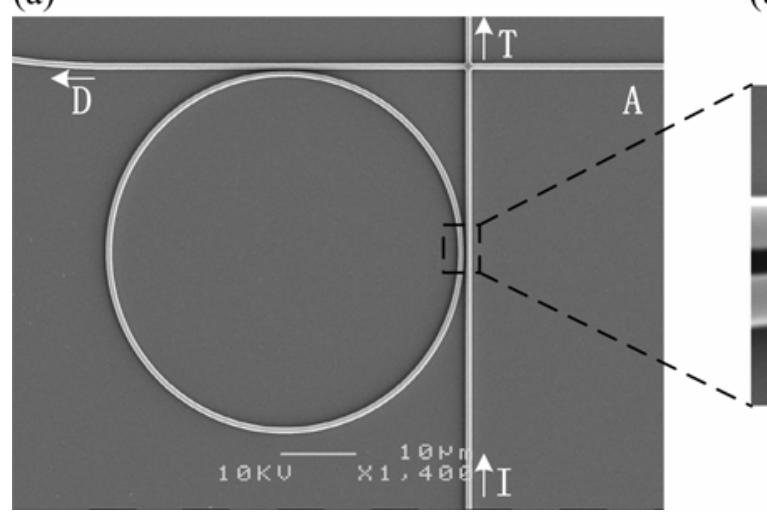

(b)

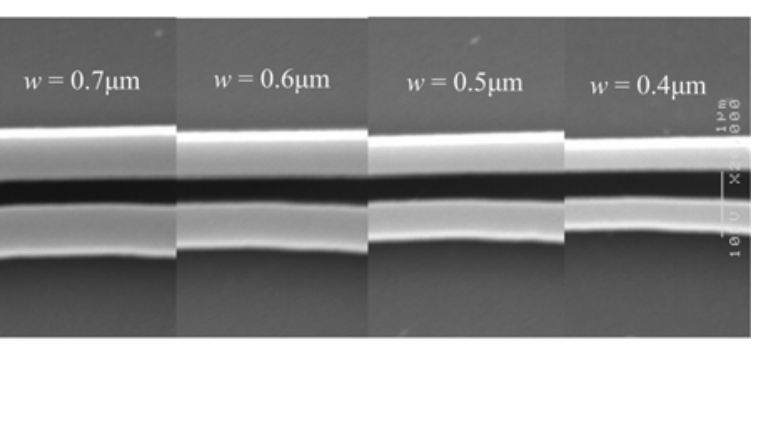

Fig. 1. (a) Scanning electron micrograph of the fabricated microring resonator-coupled waveguide crossing add-drop filter. I: Input, T: Throughput, D: Drop, A: Add. (b) Zoom-in view of the interaction region with different waveguide widths $w$ and a fixed air- gap distance. 
Figure 1 (a) shows the top-view scanning electron micrograph (SEM) of our fabricated microring-coupled waveguide crossing. The drop and add-waveguides are $90^{\circ}$ bent (with a $100 \mu \mathrm{m}$ radius of curvature) towards the throughput port direction in order to allow simultaneous measurement of all the three output ports. The waveguide crossing junction is slightly rounded (with a radius of about $0.36 \mu \mathrm{m}$ ) due to fabrication imperfections. Figure 1 (b) shows the zoom-in view SEM pictures of the waveguide-microring interaction region. The bus waveguide and the microring width $w$ simultaneously vary from about $0.7 \mu \mathrm{m}$ to about $0.4 \mu \mathrm{m}$ in $0.1 \mu \mathrm{m}$ steps. The air-gap distance remains fixed at about 0.4 $\mu \mathrm{m}$.

In order to characterize the passive filter, we employed conventional laser wavelength scanning technique as detailed in [6]. Laser light was end-fired to the input port using a tapered polarization-maintaining singlemode fiber. The chip edges are cleaved and unpolished. Figures 2 (a) -2 (d) show the measured TE-polarized (E // chip) throughput (black line) and drop (gray line) spectra of the microring-coupled waveguide crossings with different waveguide widths. The throughput and drop-port intensity are normalized with the throughput intensity of a control waveguide crossing of the same width but without a coupled microring (on the same chip). Figures 2 (e) -2 (h) show the measured side-view on-resonance microscope images of the throughput, drop and add-port for each waveguide width. The add-port intensity is weak and can be attributed to the waveguide junction scattering losses.

We remark that the normalized off-resonance throughput intensity varies from about $-5 \mathrm{~dB}$ to about 3 dB. This measured transmission variation, particularly for the higher than $0 \mathrm{~dB}$ transmission $(w=0.5 \mu \mathrm{m})$, can be attributed to the different insertion losses between the device and the control waveguide crossing. It is also worth mentioning that the control waveguide crossing has a measured transmission range from about $50 \%(w=0.5 \mu \mathrm{m})$ to exceeding $75 \%$ relative to a control straight waveguide of the same width (on the same chip). This measured transmission variation can again be attributed to the different insertion losses between the control waveguide crossing and the control straight waveguide. We note that the typical waveguide propagation loss from a similar chip [6] is about $14.8 \mathrm{~dB} / \mathrm{cm}$ (for TM polarization).

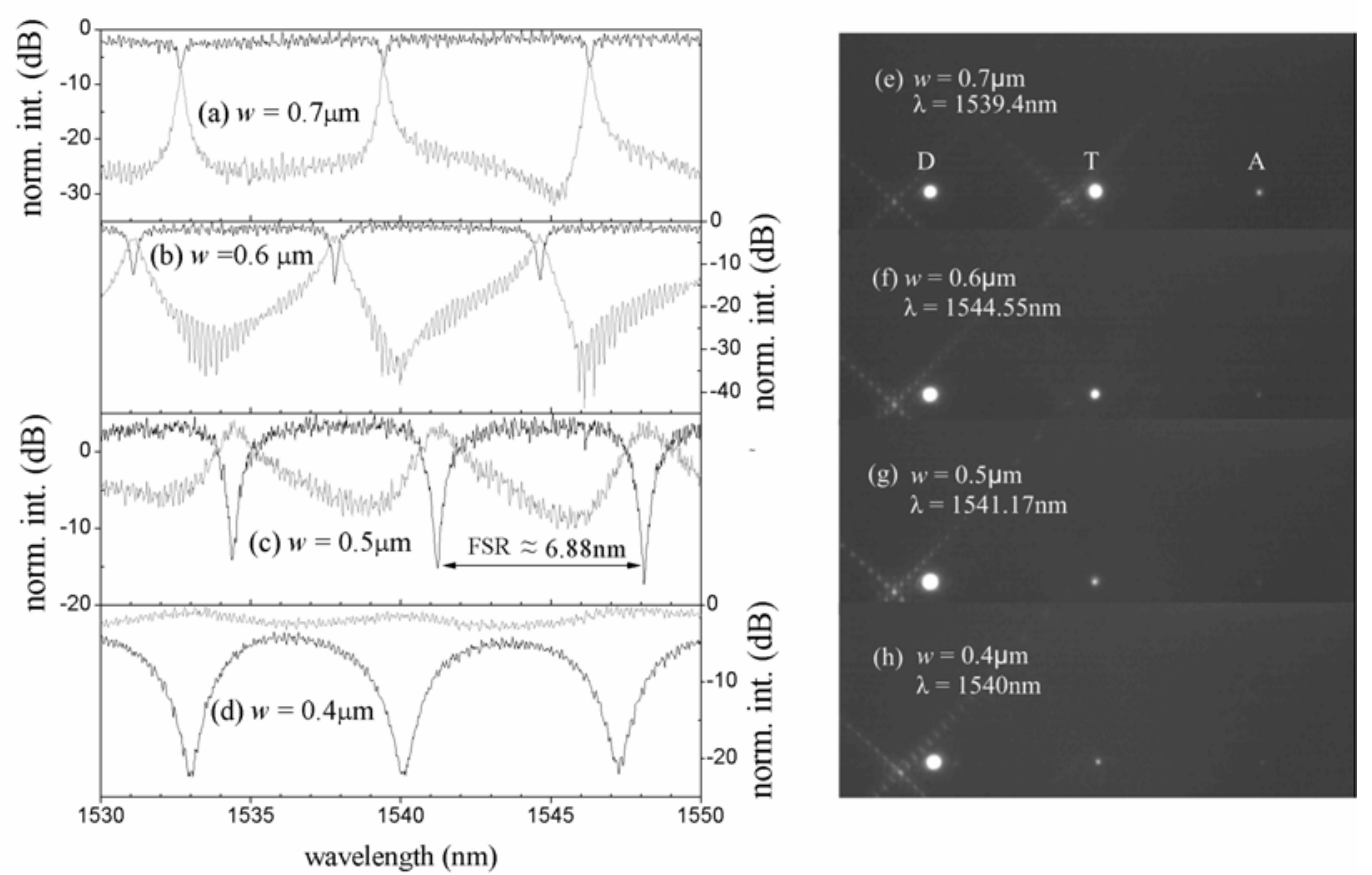

Fig.2. Measured throughput (black line) and drop (gray line) spectra of microring-coupled waveguide crossing add-drop filters. (a) $w=0.7 \mu \mathrm{m}$, (b) $w=0.6 \mu \mathrm{m}$, (c) $w=0.5 \mu \mathrm{m}$, and (d) $w=0.4 \mu \mathrm{m}$. (e) - (h) Side-view on-resonance microscope images for each $w$. D: Drop, T: throughput, A: Add

It can be seen that resonance wavelengths, coupling efficiency, peak-to-trough on-off ratio, and finesse can be tuned by varying the waveguide width. In principle, when $w$ increases, the waveguide effective 
refractive index $\left(\mathrm{n}_{\text {eff }}\right)$ increases. When $w$ increases in $0.1 \mu \mathrm{m}$ steps, the resonance wavelengths can be red-shifted (due to $\mathrm{n}_{\text {eff }}$ increases) by more than a free spectral range (FSR), which is about $6.88 \mathrm{~nm}$ in the wavelength region $1540 \mathrm{~nm}-1550 \mathrm{~nm}$. For the following analysis, we will focus on the modes whose resonance wavelengths are within this wavelength region.

Figure 3 shows the coupling efficiency and the on-off ratio as a function of the waveguide width. The highest coupling efficiency is $98.5 \%$ with $w=0.5 \mu \mathrm{m}$, and the highest on-off ratio is $20.1 \mathrm{~dB}$ with $w=0.4$ $\mu \mathrm{m}$. The smaller $w$ has a smaller $\mathrm{n}_{\text {eff, }}$, and thus less optical confinement and a better optical coupling. Figure 4 shows the waveguide width dependence of the finesse. With $w=0.7 \mu \mathrm{m}$, we obtained the maximum finesse of 28.8 (corresponding to a $\mathrm{Q} \approx 6,500$ ). The larger $w$ has a larger $\mathrm{n}_{\text {eff }}$, and thus a better optical confinement and a higher finesse.

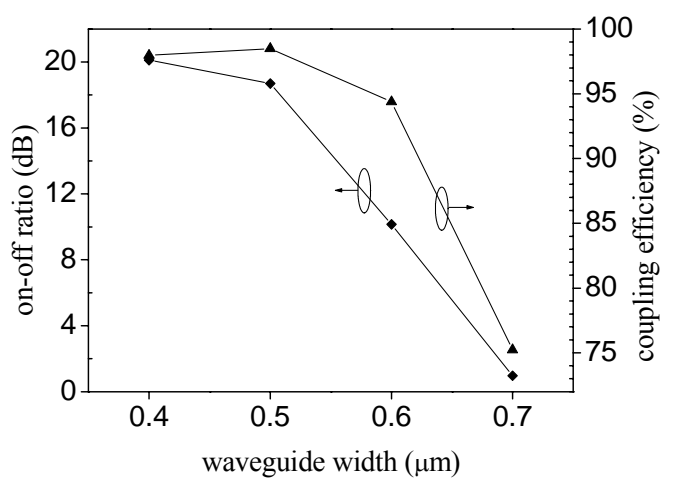

Fig. 3. Waveguide width dependence of the coupling efficiency and the on-off ratio.

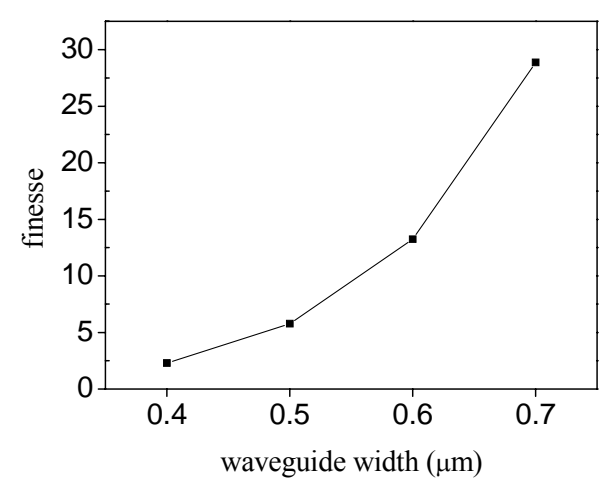

Fig. 4. Waveguide width dependence of the finesse.

In summary, we report experimentally demonstrated microring resonators laterally coupled with waveguide crossings as add-drop filters in silicon nitride. Resonances can be efficiently transferred from the input-coupled waveguide to the crossed-waveguide through the side-coupled microring. Our experiments demonstrate that resonance wavelengths, coupling efficiency, on-off ratio, and finesse can be tuned by varying the waveguide width. Our future work will focus on multiple-microring-coupled waveguide crossings in order to simultaneously route multiple wavelength channels at the same waveguide crossing junction.

The research was substantially supported by grants from the Research Grants Council and the University Grants Council of the Hong Kong Special Administrative Region, China (Project No. HKUST6166/02E \& HIA01/02.EG05).

\section{References}

[1] C. Manolatou and H. A. Haus, Passive Components for Dense Optical Integration (Kluwer Academic Publishers, 2001).

[2] B. E. Little, S. T. Chu, H. A. Haus, J. Foresi, and J.-P. Laine, "Microring resonator channel dropping filters," J. Lightwave Technol., 15, 998 (1997).

[3] D. Rafizadeh, J. P. Zhang, S. C. Hagness, A. Taflove, K. A. Stair, and S. T. Ho, "Waveguide-coupled AlGaAs/GaAs microcavity ring and disk resonators with high finesse and 21.6-nm free spectral range," Opt. Lett., 22, 1244 (1997).

[4] T. Barwicz, M. A. Popovic, P. T. Rakich, M. R. Watts, H. A. Haus, E. P. Ippen, and H. I. Smith, "Microring-resonator-based add-drop filters in SiN: fabrication and analysis," Opt. Express, 12, 1437 (2004).

[5] S. T. Chu, B. E. Little, W. Pan, T. Kaneko, S. Sato, and Y. Kokubun, “An eight-channel add-drop filter using vertically coupled microring resonators over a cross grid," IEEE Photonics Tech. Lett., 11, 691 (1999).

[6] Chao Li, Ning Ma and Andrew. W. Poon, "Waveguide-coupled octagonal microdisk channel add-drop filters," Opt. Lett., 29, 471 (2004). 